\title{
Un dilemme de formation
}

Le Masters of Arts in Teaching en Alaska

A dilemma in training. Alaska's master of arts in teaching

Marjorie Fields et David Marvel

Traducteur : Patricia Vrinat

\section{OpenEdition}

\section{Journals}

Édition électronique

URL : http://journals.openedition.org/ries/2841

DOI : $10.4000 /$ ries.2841

ISSN : 2261-4265

\section{Éditeur}

Centre international d'études pédagogiques

\section{Édition imprimée}

Date de publication : 1 décembre 1998

Pagination : 123-128

ISSN : 1254-4590

\section{Référence électronique}

Marjorie Fields et David Marvel, «Un dilemme de formation », Revue internationale d'éducation de Sèvres [En ligne], 20 | décembre 1998, mis en ligne le 17 avril 2013, consulté le 19 avril 2019. URL : http:// journals.openedition.org/ries/2841; DOI : 10.4000/ries.2841

Ce document a été généré automatiquement le 19 avril 2019

(ㄷ) Tous droits réservés 


\title{
Un dilemme de formation
}

\author{
Le Masters of Arts in Teaching en Alaska \\ A dilemma in training. Alaska's master of arts in teaching
}

\section{Marjorie Fields et David Marvel}

Traduction : Patricia Vrinat

1 Au cours des soixante dernières années, aux États-Unis, les programmes de formation pour l'enseignement en cours moyen et dans le secondaire ont subi beaucoup de changements. Les forces politiques, sociales et économiques qui gouvernent notre société, ont obligé nos écoles à évoluer ainsi que les enseignants qui y enseignent.

Les instituts de formation des maitres ont réagi en demandant une meilleure formation universitaire dans la matière enseignée et une formation pédagogique plus poussée ${ }^{1}$.

3 Il y a une quarantaine d'années, beaucoup de professeurs étaient formés dans des « teacher colleges » spécialement conçus pour cela, plutôt que dans des universités. Dans la plupart des cas, les études duraient trois ans ou moins et l'enseignant en formation apprenait d'une manière sommaire comment enseigner un certain nombre de matières, sans avoir une connaissance approfondie de l'une d'entre elles en particulier ${ }^{2}$.

$4 \mathrm{Au}$ fur et à mesure que les connaissances dans chaque discipline augmentaient, la formation des enseignants a été l'objet de vives critiques à cause de son faible niveau académique et de son manque de rigueur. Dans un premier temps, la réponse a consisté à déplacer les programmes de formation vers les universités qui avaient des cycles de quatre ans pour la licence et à augmenter le nombre de modules dans des matières spécifiques pour avoir le droit d'enseigner. Cependant, pour pouvoir passer plus de temps sur des matières particulières, le temps passé à étudier la pédagogie dans le détail était réduit en proportion pour ne pas allonger le temps d'étude ${ }^{3}$. Dans beaucoup de nos universités, il subsiste une tension entre les exigences de la discipline, d'une part, et celles de la pédagogie, d'autre part, dans la préparation à l'enseignement secondaire. 


\section{Le Master of Arts in Teaching}

5 Une solution à ce dilemme est le modèle trouvé pour le Master of Arts in Teaching (MAT). Dans ce modèle, l'étude de la matière est totalement séparée de l'étude des techniques d'enseignement. L'étudiant reste à l'université quatre ans pour préparer un Bachelor's degree traditionnel dans une matière donnée. Ensuite, il peut demander à suivre un programme de préparation à l'enseignement fondé sur le nouveau modèle du MAT. À la suite de ce programme, il obtiendra un Master's degree et l'État sera en mesure de l'autoriser à enseigner.

6 À l'université de l'Alaska du Sud-Est (UAS), le programme de MAT dure douze mois. Les candidats ont un Bachelor's degree dans une matière scolaire, avec une bonne moyenne. Très souvent, ce sont des gens qui ont cinq ans d'expérience dans le monde de travail, voire plus, et ils ont près de la trentaine. Cependant, comme le programme est mieux accepté, on voit maintenant des étudiants qui demandent à le suivre directement après leur Bachelor's, ainsi que des gens qui cherchent à avoir une deuxième carrière, après une trentaine d'années passées dans un autre emploi. Dans la classe actuelle de vingt étudiants, il y a trois stagiaires de plus de cinquante ans et quatre de moins de vingt-cinq ans.

7 En général, il y a une cinquantaine de candidats pour ce cours, mais nous n'en acceptons pas plus de vingt à cause du temps nécessaire pour surveiller leur travail en classe. Chaque étudiant est observé dans son école au moins une fois par semaine, si ce n'est plus. Les stagiaires suivent des cours sur le campus Juneau dès l'été de leur admission au programme et passent quatre jours par semaine dans des écoles et un jour à l'université pendant l'année scolaire. Puis ils passent un deuxième été sur le campus universitaire pour suivre d'autres cours.

8 Il y a deux étapes pour les candidats. Tout d'abord, les étudiants doivent montrer qu'ils ont le niveau requis pour suivre des cours après le Bachelor's degree. Ensuite, les enseignants du district examinent leurs dossiers pour voir s'ils aimeraient accueillir un des candidats dans leur classe l'année suivante. Cette coopération entre les districts scolaires et l'université est primordiale pour la réussite du programme; les recherches actuelles indiquent que les programmes de préparation à l'enseignement, qui établissent des partenariats constructifs avec les districts locaux, produisent des enseignants qui sont mieux préparés à confronter les difficultés à enseigner aux jeunes très divers d'aujourd'hui ${ }^{4}$. S'il n'y avait pas d'enseignants volontaires pour être tuteur de nos étudiants, le programme n'existerait pas

9 La mission du MAT est de préparer les étudiants à une carrière d'enseignant dans nos écoles publiques, plus particulièrement en Alaska. Chaque année, les districts scolaires de l'Alaska embauchent plus de cinq cents enseignants. 


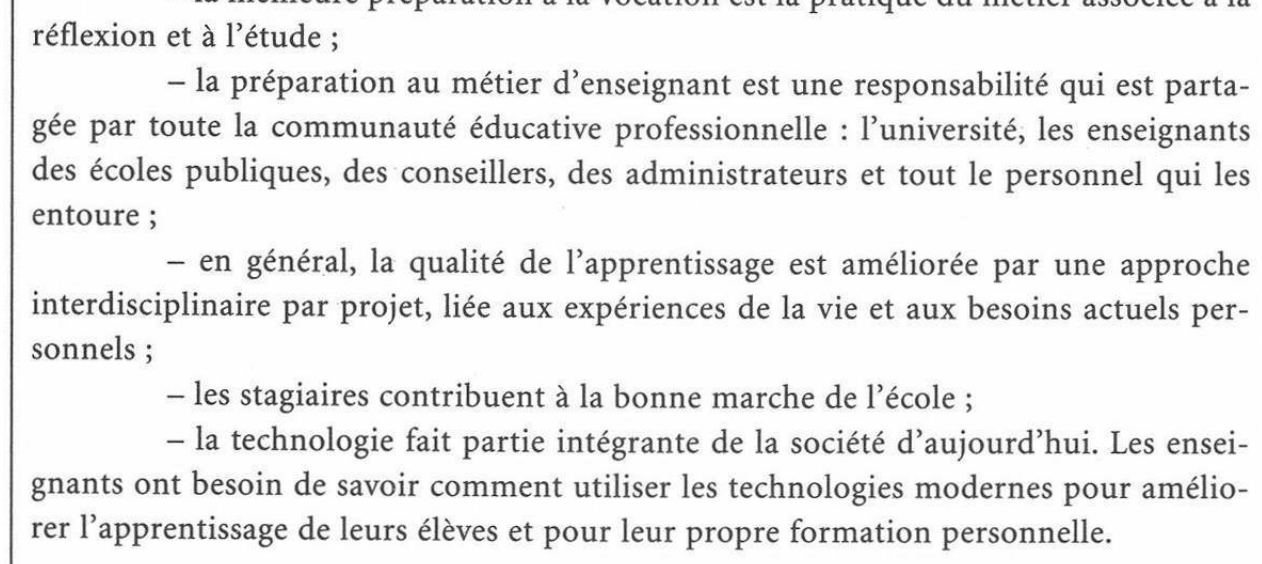

- la préparation au métier d'enseignant est une responsabilité qui est partagée par toute la communauté éducative professionnelle : l'université, les enseignants des écoles publiques, des conseillers, des administrateurs et tout le personnel qui les entoure ;

- en général, la qualité de l'apprentissage est améliorée par une approche interdisciplinaire par projet, liée aux expériences de la vie et aux besoins actuels personnels ;

- les stagiaires contribuent à la bonne marche de l'école ;

- la technologie fait partie intégrante de la société d'aujourd'hui. Les enseignants ont besoin de savoir comment utiliser les technologies modernes pour améliorer l'apprentissage de leurs élèves et pour leur propre formation personnelle.

- la meilleure préparation à la vocation est la pratique du métier associée à la

Parmi ces nouveaux recrutés, environ $70 \%$ sont des enseignants qui viennent d'un autre État, avec très peu, voire aucune connaissance des problèmes complexes auxquels les élèves et les éducateurs de l'Alaska sont affrontés.

11 Le MAT pour l'enseignement secondaire met l'accent sur la formation d'enseignants avec un bon niveau dans leur discipline d'enseignement (c'est-à-dire un bachelor's degree dans la discipline enseignée) ; un programme de formation post-gradué exigeant; une année d'expérience de la classe qui sert de base de recherche et de réflexion.

\section{Les objectifs de compétences à l'université du Sud-Est de l'Alaska ${ }^{5}$}

\section{La compétence en communication}

Les élèves qui quittent le lycée devraient savoir écrire, parler, lire et écouter efficacement.

L'enseignant engage un examen critique et réfléchi de ses pratiques avec d'autres. Il met en application, comme il convient, ses connaissances sur l'histoire de l'Alaska, la géographie, l'économie, la vie politique, les langues, les modes de vie traditionnels et l'actualité, en choisissant ses stratégies éducatives, ses matériaux et ses ressources. Il identifie les étapes de développement nécessaires à la maîtrise des contenus de son enseignement et applique les méthodes qui conviennent pour évaluer le niveau des élèves dans une matière donnée ; il met aussi en pratique les stratégies appropriées, $\mathrm{y}$ compris la collaboration avec d'autres, pour faciliter les progrès de l'élève. Il communique les compétences et les concepts de base des différents champs disciplinaires avec exactitude. Il vise un bon niveau pour les performances de ses élèves et exprime clairement les attentes par rapport à ce que l'élève apprend. Il encourage et entretient des communications constructives et régulières entre la classe et les familles des élèves. Il participe à tous les efforts entrepris par l'école pour communiquer avec la communauté qui l'entoure et pour impliquer les parents dans l'apprentissage des élèves. Il fait le lien, par ses méthodes d'enseignement, entre l'école et les activités de classe, les familles et leurs cultures, ainsi qu'avec le monde du travail et la communauté. Il fait participer les parents quand il s'agit de 
fixer et de suivre les objectifs d'un élève. Il communique, travaille en coopération et développe des relations professionnelles avec d'autres collègues.

\section{La compétence dans le raisonnement mathématique}

Une personne qui maitrise le calcul est capable de raisonnement et d'analyse mathématiques.

L'enseignant crée, sélectionne, adapte et utilise toute une série de méthodes d'évaluation qui permettent d'avoir des renseignements sur l'apprentissage des élèves, qui le renforce et aide les élèves, les parents, les administrateurs et toute autre personne concernée. Il réfléchit sur les renseignements apportés par ces évaluations et adapte ses pratiques d'enseignement en conséquence, pour faciliter les progrès de l'élève et pour qu'il atteigne les objectifs fixés dans les programmes.

\section{Compétence dans la compréhension des informations}

Cette compétence combine plusieurs compétences : savoir identifier, accéder à..., analyser et synthétiser des informations.

\section{Compétence dans le comportement professionnel}

On attend des élèves qu'ils adoptent un certain comportement professionnel en classe. Leur succès dans la vie professionnelle dépend de plusieurs types de comportement.

L'enseignant engage un examen critique et réfléchi de ses pratiques avec d'autres. Il pose pour principe la dignité et la valeur de tous les élèves, en favorisant une atmosphère d'équité ainsi que la diversité. Il fait preuve de ses connaissances de la structure académique du champ disciplinaire enseigné, de ses outils et de ses méthodes de recherche, des concepts de base et des liens possibles avec d'autres domaines de connaissances. Il reste au courant de son champ disciplinaire et fait le lien entre cette matière et ses applications dans la salle de classe, la vie de travail et la communauté. Il communique les compétences et les concepts de base des différents champs disciplinaires avec exactitude. Il montre de l'enthousiasme pour le contenu des cours. Il crée et entretient une communauté scolaire stimulante, sans exclusions, dans une atmosphère de sécurité, dans laquelle les élèves acceptent de prendre des risques intellectuels, et travaillent de manière indépendante mais aussi en collaboration. Il établit des règles de conduite claires et surveille le comportement des élèves. Il travaille en collaboration avec les parents et les familles pour favoriser et promouvoir l'apprentissage de l'élève. Il fait preuve d'une éthique professionnelle irréprochable. Il entretient et réactualise à la fois le contenu du champ disciplinaire et les meilleures pratiques pédagogiques. Il entreprend des activités instructives pour le développement de la qualité des programmes qui concernent la vie de la classe, de l'école, ou du district scolaire. Il communique, travaille en coopération et développe les relations avec d'autres collègues.

\section{Compétence en études multiculturelles}

L'enseignant met en pratique les théories sur l'apprentissage, prend en compte les différentes façons d'apprendre de ses élèves, y compris les différences d'intelligence, 
de perception et de style cognitif. Il inclut des caractéristiques de la culture familiale et locale dans des stratégies d'enseignement qui facilitent l'apprentissage des élèves. Il identifie et utilise des méthodes d'enseignement et les ressources qui conviennent aux besoins individuels et spécifiques des élèves. Il met en application, comme il convient, ses connaissances sur l'histoire de l'Alaska, la géographie, l'économie, la vie politique, les langues, les modes de vie traditionnels et l'actualité, dans le choix de ses stratégies éducatives, de ses matériaux et de ses ressources. Il pose pour principe la dignité et la valeur de tous les élèves, en favorisant une atmosphère d'équité ainsi que la diversité. Il fait le lien, par ses méthodes d'enseignement, entre l'école et les activités de classe et les familles et leurs cultures, et avec le monde du travail et la communauté.

\section{Compétence dans la théorie de l'éducation}

L'enseignant cerne, avec précision, les capacités de développement de ses élèves et adapte son enseignement en conséquence. Il met en pratique les théories sur l'apprentissage, prend en compte les différentes façons d'apprendre de ses élèves, $y$ compris les différences d'intelligence, de perception et de style cognitif. Il identifie les étapes de développement nécessaires à la maîtrise des contenus de son enseignement, et applique les méthodes qui conviennent pour évaluer le niveau des élèves dans une matière donnée ; il met aussi en pratique les stratégies appropriées, y compris la collaboration avec d'autres, pour faciliter les progrès de l'élève. Il organise et donne un enseignement qui correspond aux caractéristiques de ses élèves et aux objectifs du programme. Il planifie et utilise une variété de techniques d'organisation de la classe pour établir et entretenir une atmosphère qui permet à tous les élèves d'apprendre. Il les aide à accepter leur propre responsabilité dans le processus d'apprentissage. 


\begin{tabular}{|c|}
\hline Stage \\
\hline $\begin{array}{l}\text { Le stage à plein temps de la maîtrise d'enseignement donne une année entière de pra- } \\
\text { tique à l'étudiant sur le lieu de travail. Les résultats de l'étudiant sont évalués par le tuteur uni- } \\
\text { versitaire, l'enseignant formateur et par l'auto-évaluation de l'étudiant. Les critères d'évaluation } \\
\text { sont en accord avec les objectifs de compétences et de connaissances établis par le département } \\
\text { universitaire. }\end{array}$ \\
\hline Auto-évaluation de première année \\
\hline $\begin{array}{l}\text { Un formulaire d'évaluation est envoyé à chaque enseignant nouvellement diplômé au } \\
\text { cours de sa première année d'enseignement. Les données collectées sont classées avec le formulai- } \\
\text { re d'évaluation du chef d'établissement (voir ci-dessus), des statistiques sont faites et inclues dans } \\
\text { un rapport annuel. }\end{array}$ \\
\hline Évaluation de première année par le chef d'établissement \\
\hline $\begin{array}{l}\text { Un formulaire d'évaluation est envoyé au chef d'établissement de chaque nouvel ensei- } \\
\text { gnant. On lui demande de critiquer l'enseignant de première année sur la même base de compé- } \\
\text { tences et de connaissances que celle utilisée tout au long du programme de formation. Ces infor- } \\
\text { mations sont classées avec l'auto-évaluation du nouveau diplômé. }\end{array}$ \\
\hline Auto-évaluation de troisième année \\
\hline $\begin{array}{l}\text { Un formulaire d'évaluation est envoyé à chaque diplômé au cours de sa troisième année } \\
\text { d'enseignement. Les données collectées sont classées avec le formulaire d'évaluation du chef d'éta- } \\
\text { blissement (voir ci-dessous), des statistiques sont faites et incluses dans un rapport annuel. }\end{array}$ \\
\hline Évaluation de troisième année par le chef d'établissement \\
\hline $\begin{array}{l}\text { Un formulaire d'évaluation est envoyé au chef d'établissement de chaque enseignant. } \\
\text { On lui demande de critiquer l'enseignant de troisième année sur la même base de compétences et } \\
\text { de connaissances que celle utilisée tout au long du programme de formation. Ces informations } \\
\text { sont classées avec l'auto-évaluation de l'étudiant. }\end{array}$ \\
\hline
\end{tabular}

Procédés et instruments d'évaluation du programme de formation

\section{Rencontres avec les enseignants formateurs}

Le personnel du programme du MAT rencontre les enseignants formateurs régulièrement, à la fois séparément et en groupes, pour discuter du programme et de son efficacité.

\section{Rencontres avec les principaux}

Les responsables du programme de formation rencontre les chefs d'établissement régulièrement, à la fois séparément et en groupes, pour discuter du programme et de son efficacité.

\section{Réunions du département de formation des enseignants}

On discute du programme de formation et on le révise régulièrement lors de réunions mensuelles du département.

\section{Groupe de conseil du département de formation des enseignants}

$\mathrm{Au}$ groupe de conseil siègent des représentants des écoles de Juneau, à la fois enseignants et administrateurs, et des représentants du département d'éducation de l'État d'Alaska. Le groupe se rencontre une fois par mois pour débattre des programmes de formation, et pour conseiller l'université et le doyen de l'université sur tout ce qui concerne leur conception et leur mise en place.

\section{Conseil des diplômes de l'UAS}

Le rôle principal de ce conseil est de contrôler et d'approuver les formations offertes et les programmes de l'université. C'est à ce titre que le conseil donne son avis à la faculté responsable de la formation et qu'il approuve tout changement de programme. 


\section{NOTES}

1. C. Reynolds (Ed), Knowledge Base for the Beginning Teacher, New-York, Pergamon Press, 1989.

2. J. I. Goodlad, S. Soder, K. A. Sirotnik (Eds), Places where Teachers are Taught, San Francisco, Jossey -Bass, 1990.

3. J. I. Goodlad, Teachers for our Nation's Schools, San Francisco, Jossey-Bass, 1990.

4. L. Darling-Hammon, The Right to Learn: a Blue Print for Creating Schools that Work, San Francisco, Jossey-Bass, 1997.

5. Certaines de ces capacités relèvent d'une approche transversale et se retrouvent dans plusieurs pôles de compétences.

\section{RÉSUMÉS}

Le Master of Art in Teaching prépare à l'enseignement dans l'État de l'Alaska en un an. Les candidats doivent faire preuve d'un bon niveau dans leur discipline pour avoir accès à la formation qui comprend des études théoriques et des stages. Un système d'évaluation de l'enseignant stagiaire et de l'enseignant en fonction permet d'améliorer et de faire évoluer constamment cette formation. Les objectifs de compétence présentés étonneront plus d'un lecteur européen.

The Master of Arts in Teaching is a one year training course for teaching in the State of Alaska. Candidates must be of a good level in their subject to be able to follow the course which involves both theoretical studies and training periods. A system of assessment of the trainee teacher and the teacher instructing him makes constant improvements in training possible. The aims of competence presented will come as a surprise to many an European reader.

\section{INDEX}

Index géographique : Canada, Alaska

Mots-clés : formation des enseignants, enseignant, évaluation de l'enseignant

\section{AUTEURS}

\section{MARJORIE FIELDS}

Coordinator of Early Childhood Teacher Education, University of Alaska Southeast, Juneau, États-Unis

\section{DAVID MARVEL}

Coordinator of Secondary Level Teacher Education, University of Alaska Southeast, Juneau, États-Unis 